This ephemeris shows the value, $z$, of the invisible line of sight coordinate, as well as the rate, $\Delta$, at which this increases in four years. This latter quantity is required by spectroscopists, who wish to determine the parallax by comparing $\Delta$ with the line of sight velocity expressed in diameters of the earth's orbit per annum. Owing to the moderate value of the inclination of the orbit of $\sigma$ Coronae it is perhaps not likely that the latter quantity will be measured for some years, but I am computing the same data for other binary stars. Taking the hypothetical parallax into account hints may thus be obtained as to which binary stars are most suitable for observing the difference between the line of sight motion of the two components. The hypothetical parallax is equal to 0.064 but the actual parallax is probably smaller, and the mass of this system is probably greater than the mass of the sun.

Hongkong Observatory, I905 May I $4 .^{2}$

W. Doberck.

\title{
Observations of Jupiter's sixth satellite
}

from photographs taken with the 30 inch reflector of the Thompson equatorial at the Royal Observatory, Greenwich.

\begin{tabular}{|c|c|c|c|c|c|c|c|}
\hline \multirow{2}{*}{\multicolumn{2}{|c|}{1905}} & \multirow{2}{*}{ Gr. M. T. } & \multirow[b]{2}{*}{$p$} & \multirow{2}{*}{$s$} & \multicolumn{2}{|c|}{ Tab.-Obs. $\left.{ }^{*}\right)$} & \multirow{2}{*}{ Exp. } \\
\hline & & & & & & & \\
\hline Aug. & 23 & I $3^{\mathrm{h}} 30^{\mathrm{m}}$ & $3 \mathbf{I} \mathbf{1}^{\circ} \quad 9^{\prime}$ & $25^{\prime} 30^{\prime \prime}$ & - & - & $30^{\mathrm{m}}$ \\
\hline & 23 & 1423 & 31045 & 2533 & - & - & $3 \mathbf{I}$ \\
\hline \multirow[t]{8}{*}{ Sept. } & 3 & 15 I 7 & $290 \quad 5^{I}$ & $3^{6} 59$ & $+4^{\circ}: 8$ & -0.5 & 40 \\
\hline & 7 & I3 26 & $286 \quad 54$ & $40 \quad 5^{2}$ & +3.9 & $-\mathbf{I} .0$ & 60 \\
\hline & 7 & I 532 & $286 \quad 48$ & $40 \quad 54$ & +4.0 & -1.0 & 55 \\
\hline & 8 & I 256 & $285 \quad 54$ & $4 \times 43$ & +3.7 & -0.9 & 30 \\
\hline & 12 & I4 37 & $28 I \quad 29$ & $45 \quad 16$ & +4.0 & $-\mathrm{r} .5$ & 20 \\
\hline & 12 & I $5 \quad 5$ & $281 \quad 26$ & 45 I7 & +4.0 & -1.5 & 20 \\
\hline & 12 & 1542 & $28 I, 32$ & $45 \quad$ I 7 & +4.0 & - I. 5 & 30 \\
\hline & 30 & 1217 & 26734 & $54 \quad 53$ & +3.2 & -0.6 & 60 \\
\hline \multirow[t]{3}{*}{ Oct. } & 4 & 1225 & $2645 \mathrm{I}$ & $55 \quad 29$ & +2.8 & -0.5 & 40 \\
\hline & 4 & 1638 & 2644 I & $553 \mathrm{I}$ & +2.9 & -0.5 & 39 \\
\hline & 5 & II $5^{2}$ & $264 \quad 3$ & 5533 & +2.7 & -0.5 & $3^{\circ}$ \\
\hline
\end{tabular}

The photographs were taken by Mr. Melotte, with the exception of two taken by Mr. Edney (Sept. 8 and Oct. 4) and two taken by Mr. Davidson (Oct. 5).

Supplementary exposures were given on several of the plates for determination of orientation, and the position angles were inferred from these by reference to a pair of stars common to all the plates.

The over-exposed image of Jupiter was reduced with ferri-cyanide of potassium, leaving a reversed image which was easily measurable.

The measures of position angle and distance from Jupiter, made in the position micrometer, are: to be considered as merely provisional pending more complete measures with the help of reference stars. No corrections have as yet been applied for differential refraction or aberration.

*) Vgl. die Ephemeride von F. E. Ross A. N. 4042. $K r$.

\section{Elementi del pianeta (554) Peraga.}

Il pianeta Peraga scoperto da P. Götz a KönigstublHeidelberg l' 8 Gennajo I905 fu osservato in questa prima apparizione, per quanto è a mia conoscenza, ad Arcetri, Düsseldorf, Padova, Roma, Vienna. Con le tre osservazioni fatte a Padova all' equatoriale Dembowski, due dal prof. Antoniazzi nelle notti ${ }_{3} 3$ Gennajo e 24 Febbrajo ed una da me nella notte del 3 Febbrajo, seguendo il metodo di Encke-Tietjen, ho ottenuto questi elementi :

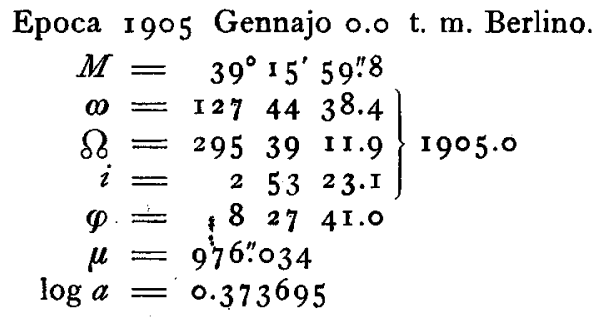

Una effemeride calcolata su questi elementi per l' intervallo di tempo dal ro Gennajo al 13 Marzo $1905 \mathrm{mi}$ ha permesso di calcolare le differenze $\mathrm{O}-\mathrm{C}$ in ascensione retta ed in declinazione per ciascuna delle $5^{8}$ osservazioni ese- guite nel predetto intervallo e nei luoghi sopra indicati. Tali differenze furono raggruppate nei tre seguenti luoghi normali fondati rispettivamente sopra 18,22, r 8 osfer 8 azioni considerate tutte come di uguale peso:

\begin{tabular}{|c|c|c|c|c|c|c|}
\hline \multirow{2}{*}{$\begin{array}{l}\text { T. m. Berlino } \\
\text { 1905 }\end{array}$} & \multicolumn{2}{|c|}{$\mathrm{O}-\mathrm{C}$} & \multicolumn{4}{|c|}{1905.0} \\
\hline & $\Delta \alpha$ & $\Delta \delta$ & & $\alpha$ & & $\delta$ \\
\hline $7 \cdot 5$ & -0.46 & - 0.8 & $8^{\mathrm{h}}$ & $51 \div 96$ & $+18^{\circ}$ & $5 I^{\prime} 25^{\prime \prime} 8$ \\
\hline Febb. 8.5 & +0.33 & -3.7 & 8 & I 1.84 & +19 & $25 \quad 37.9$ \\
\hline Marz. 6.5 & -2.17 & $+5 \cdot 3$ & 750 & I 5.65 & +19 & $28 \quad 7.1$ \\
\hline
\end{tabular}

Partendo da questi tre luoghi ho ricalcolato l'orbita ed ho ottenuto gli elementi eclittici:

Epoca I905 Gennajo 0.0 t. m. Berlino.

$$
\begin{aligned}
& M=4 \mathrm{I}^{\circ} 20^{\circ} \mathrm{I} 5^{\prime \prime} 3 \\
& \omega=\begin{array}{lll}
124 & 25 & 29.3
\end{array} \\
& \delta=29543 \quad 16.2 \text { I905.0 } \\
& i \Longrightarrow 256 \text { 13.2 } \\
& \varphi=85453.0 \\
& \mu=969 \text { ". } 64 \\
& \log a=0.37574^{\circ}
\end{aligned}
$$

